\title{
Health information needs and information seeking behavior of pregnant women attending antenatal clinics of Jaffna Teaching Hospital
}

\author{
K. Murugathas ${ }^{1}$, T. Sritharan ${ }^{2}$, S. Santharooban ${ }^{3}$
}

\begin{abstract}
Pregnant women face many challenges during the pregnancy period. They need quality, reliable and easily accessible information in order to face the challenges. The aim of this study was to identify the information needs and information-seeking behaviour of pregnant women attending antenatal clinics of Jaffna teaching hospital. The descriptive survey research strategy was used in the study. The data was collected by using intervieweradministered questionnaire. A sample of four hundred pregnant women was selected from the pregnant women attending antenatal clinics of Jaffna teaching hospital using a systematic sampling technique. Collected data from the questionnaires were analyzed using quantitative techniques in SPSS software. Correspondence analysis was performed to find out the association between the information sources that pregnant mothers used and other study variables. Majority of the pregnant women (61\%) searched for information either always or often. Family members and friends were the main sources of information used by the greatest number of pregnant women (63\%). It was followed by public health midwives (61\%) and doctors (49\%). Internet was also identified as one of the sources of information used by $29 \%$ of the participants. Participants showed less interest in mass media, books and journals. The most needed types of information were pregnancy complications $(55.8 \%)$, delivery complications (54\%), methods of child delivery (52\%) and special tests required during pregnancy (48\%). The least important type of information was exercise during pregnancy. Unavailability of information was the most restricting barrier in accessing information.
\end{abstract}

1 Senior Assistant Librarian, Medical Library, University of Jaffna, Sri Lanka.

Email: komathym@univ.jfn.ac.lk (iD) https://orcid.org/0000-0002-7252-0342

2 Senior Assistant Librarian, University of Colombo, Sri Lanka.

Email: sritharan@lib.cmb.ac.lk

3 Senior Assistant Librarian, Faculty of Health Care Sciences, Eastern University of Sri Lanka. Email: santharoobans@esn.ac.lk 
experienced by pregnant women. It was followed by language barrier and financial status of pregnant women. The study showed that there was a significant association between the educational level of pregnant women and the information sources they preferred $\left(x^{2}=96.3, \mathrm{p}=0.00\right)$. Further it revealed that there was a significant association between age group and the information sources they often used $\left(x^{2}=57.7, p=0.03\right)$. Publishing more information in native languages of the pregnant women on web based and printed media is suggested to improve the easy accessibility of health information. The findings of the study would be useful in organizing health education programs and efficient information systems to satisfy the information needs of pregnant mothers.

Keywords: Information Needs, Information Seeking Behavior, Pregnant Women, Information Sources, Health Information 


\section{Introduction}

Pregnancy is a vital transition and precious period in any women's life. Pregnant women undergo physiological, psychological and social changes throughout the pregnancy period. During this period, the health related information is vital since the decisions and changes would affect the mother as well as the unborn fetus, who are the future citizens of a country. The health of a baby starts from the womb of the mother. Healthcare of a pregnant woman directly reflects the healthiness of her baby, which has an impact on the creation of a healthy nation in the country. One of the healthcare programs delivered to pregnant mothers was providing pregnancy related information. For this activity to become effective, it is better to study the information-seeking behavior of pregnant mothers.

Wilson (1999) defines the term information seeking behavior as "the totality of human behavior in relation to sources and channels of information, including both active and passive information seeking and information usage". According to Wilson (2000), the individual may interact with manual information in the form of a newspaper or a library or with computer-based systems such as the web. Various published books are available only to educated people especially in developing countries. In order to ensure safe delivery of the baby and to live a healthy life during and after the pregnancy, pregnant women must be provided necessary information.

As a potential mother, she may experience differed changes in her physical behaviors, health needs, health information needs, as well as in the level of nutrition of food for her and the unborn fetus. In order to face all these challenges, proper information sources are very essential during pregnancy. Self-care of a pregnant woman is very important to make decisions for the health of the mother and the fetus. Maternal mortality rate and infant mortality rate are included among the health indicators when measuring the development of a country. Nowadays people are exposed to a wide variety of information sources. It is very important to make available or accessible the timely and reliable information the pregnant women need. So far no research study has been conducted in Sri Lanka to find out the information needs and the information seeking behavior of pregnant women. By investigating the information seeking behavior of pregnant women, improvements can be 
made in the process of providing relevant information sources for pregnant women. A study on Information needs is very important if it is planning to provide an effective information service to meet the needs of the particular group. The findings of the study would be helpful to plan antenatal health educational programs in this region and to encourage the mothers to actively participate in those and learn their responsibilities. Further, it will be helpful to organize proper information services for pregnant women which would help to improve maternal and infant health.

\section{Objectives of the study}

The main objective of the study is to analyze the information needs and information seeking behavior of pregnant women who are attending antenatal clinics of Teaching hospital, Jaffna.

The specific objectives are:

i. To identify the information needs of pregnant women.

ii. To analyze the information seeking behavior of pregnant women

iii. To identify the barriers affecting the effective use of information.

iv. To determine the relationship between the demographic factors and their information needs.

\section{Literature Review}

User studies are valuable in the field of Library Science. The real beginning of the anxiety of the information seeking behavior was in the Royal Society which was held in 1948 especially with the subject interest of Science and Technology (Wilson, 2000).

With regard to the study on sources of information used by pregnant women, Aaronson, Mural and Pfoutz, (1988) identified health care providers and published books were the main sources of information. Further, the study analyzed the association between their socio-economic status and the type of information they used during pregnancy. It concluded that pregnant women with high socio-economic status mainly depended on books than women with low socio-economic status who depended on verbal communication. A study on minority ethnic women in UK revealed that many pregnant women sought information from general practitioners and healthcare visitors for 
maternity information and verbal information was preferred by pregnant women (Davies and Bath, 2002). More than half of pregnant women acquired health information by using the internet especially Google and other health related tools to find out information. YouTube, commercial pages and women forums were the information sources used by these pregnant women (Almoajel and Almarqabi, 2016)

Durham (1988) conducted a study in Wellington hospital in New Zealand and the study identified the unmet needs of women on a special test done on pregnancy, post-natal care, caesarean section and induction of labour. Singh and Newburn (2000) identified the unmet needs among pregnant women. It revealed that the mothers having the first baby were not satisfied with available information and they felt that too little information was available for them about the pregnancy. It further suggested that pregnant women needed up-to-date information, commonly asked problems and answers, solutions with various options describing the advantages and disadvantages of each of them.

When considering the language barrier in accessing information, nonEnglish speaking women in Australia were less likely preferred written and web-based documents (Grimes, Forster and Newton, 2014). The information needs of the non-English speaking women were not met with the available information sources because they were unable to use the multilingual information sources. The study concluded that for more than $50 \%$ of the participants mentioned that the internet did not play a major role in providing information to pregnant women. Internet was identified as one of the preferred information sources among pregnant women in Iran (Kamali, Ahmadian and Bahaadinbeigy, 2017). Family members were the main source of information and internet was rarely used to meet their information needs (Song et al., 2013).

Bianchi et al., (2016) conducted a qualitative study on concerns, attitudes, beliefs and information seeking practices with respect to nutrition related issues during pregnancy. In this study, it was found that pregnant women in France get the nutrition information from healthcare providers. Most of the women were interested to access information on fetal development and 
stages during the pregnancy. Onuoha and Amuda (2013) conducted a study on information seeking behavior of pregnant women in selected hospitals of Ibadan metropolis. In this study doctors and nurses were identified as the most available sources. This study recommended making the availability of health information sources and libraries at the government hospitals for pregnant women.

\section{Methodology}

The descriptive survey research method was used in this study. The study was conducted in the antenatal clinics of Jaffna Teaching hospital. The population of the study was pregnant women attending antenatal clinics of Jaffna Teaching hospital. There were three clinics per week. Based on the records available in the hospital, there are about 80 pregnant women attending each clinic per day. A sample size of 384 pregnant women was calculated by using Cochran's formula at $50 \%$ of the variability and $95 \%$ confidence interval with $\pm 5 \%$ precision (Cochran, 2007). A systematic sampling of 400 pregnant women was selected for the study in order to reduce the sampling error of the study. Every fourth woman was selected for the study from the registration number of the clinic on the day. If the mother refused to participate in the study, the next mother was considered. The study was continued until it reached the required sample size. Written consent was taken from all the participants of the study.

An interviewer administered questionnaire was used as the research instrument to collect the data. The questionnaire was developed based on the validated questionnaires (Kamali, 2017; Grimes et al., 2014; Almoajel \& Almarqabi, 2016). The questionnaire consists of both structured and a few open ended questions. The questionnaire has four parts mainly covering demographic information of pregnant women, and their information needs, information sources they were using and barriers in accessing the information sources. The questionnaire was translated in to Tamil and Sinhala language. The questionnaire was pilot tested among pregnant women in a preliminary study sample size of thirty in order to find out the suitability of the questionnaire for the study. Those who were involved in the pilot study were excluded from the sample. The questionnaire was modified based 
on the results of the preliminary study. The data was collected during the period from February 2019 to June 2019.

Collected data from the questionnaire was analyzed with SPSS software using descriptive and inferential statistics. The correspondence analysis was performed to find out the association between the information sources that pregnant mothers used and other study variables such as the age group, educational level and family structure. The correspondence analysis is similar to chi-squared test but it is often used for high dimensional contingency tables. Ethical clearance to carry out the study was obtained from the ethical review committee, Faculty of Medicine, University of Jaffna (Reference Number: J/ERC/18/93/NDR/0194).

\section{Results and Discussion}

Socio-demographic characteristics of the participants are given in Table 1. The age of the majority of the respondents in the study was in the range of $26-35$ years $(n=222,55 \%)$. About $9 \%(n=37)$ of the participants were less than twenty years and $3 \%(n=12)$ of the participants were above forty years. Table 1 indicates that about $54 \%(n=216)$ of the participants attained at least advanced level education or above. The majority $(60 \%, n=239)$ of the study participants were housewives. It was reported that average monthly household income of $65.3 \%(n=261)$ of the study participants was less than 50,000 Rupees. Majority of the selected study sample $(87 \%, \mathrm{n}=348)$ comprised of a first or second pregnancy. Only $12.8 \%(n=51)$ was a third pregnancy or above. Thirty three percentage $(n=130)$ of the mothers were experiencing the first trimester whereas $45.5 \%(n=182)$ of them were in their third trimester of pregnancy.

Table 1. Socio demographic characteristics of participants and pregnancy information.

\begin{tabular}{|c|c|c|}
\hline Demographic characteristics $(n=400)$ & $\begin{array}{l}\text { Frequency } \\
\text { (n) }\end{array}$ & Percentage $(\%)$ \\
\hline \multicolumn{3}{|l|}{ Age } \\
\hline$<20$ years & 37 & 9.3 \\
\hline $21-25$ years & 78 & 19.5 \\
\hline $26-30$ years & 103 & 25.8 \\
\hline $31-35$ years & 119 & 29.8 \\
\hline
\end{tabular}




$$
\begin{aligned}
& \text { 36-40 years } \\
& >40 \text { years }
\end{aligned}
$$

\section{Highest education attained}

Grade 5 and below

Up to $\mathrm{O} /$ Level

Up to A/Level

Graduate

Postgraduate and above

\section{Occupation}

Employed

House wife

Monthly average Family income

\section{( Sri Lankan Rupees)}

$$
>20,000
$$

$20,000-50,000$

Above 50,000 -100,000

$>100,000$

Family structure

Nuclear family

Extended family
77

184

69

70

192

208
12

12.8

23.3

93

30.5

1

0.3

157

40

239

60
19.3

46

17.3

17.5

48

52

\section{Number of pregnancy}

First pregnancy

177

44.3

Second pregnancy

171

42.8

Third pregnancy or above

\section{Gestation period}

First trimester

130

Second trimester

Third trimester

182

45.5

Source: Survey data

Participants were requested to report how often they needed information. The study revealed that $38.6 \%(n=152)$ of mothers always needed information, whereas $36 \%(n=142)$ needed sometimes. Twenty percent $(n=76)$ needed often and $5.6 \%$ reported they needed rarely. The majority of the participants $(61 \%)$ indicated that they needed information either always $(42 \%)$ or often $(19 \%)$. It clearly indicates that pregnant women are in need of health information during their pregnancy period. 


\section{Types of information pregnant women need}

Participants were asked to choose from twenty types of information with regard to pregnancy and child birth. They could select more than one answer. In addition there was one option for any other as an open answer. Participants were given the opportunity to select the types of information they were in need mainly with regard to pregnancy and childbirth.

Table 2 summarizes the types of information needed by pregnant mothers during pregnancy. The most needed types of information among the participants were pregnancy complications (55.8\%, $\mathrm{n}=223)$, delivery complications ( $54 \%, \mathrm{n}=216)$, methods of child delivery $(52 \%, \mathrm{n}=208)$ and the special tests required during pregnancy (48 5, $\mathrm{n}=193)$. The least important type of information as indicated by the respondents was the exercise during pregnancy $(17 \%, \mathrm{n}=69)$. This may be due to their lack of knowledge that they were not aware of the importance of exercise during pregnancy. They had less interest in the topics of physical and psychological changes after delivery $(18 \%, \mathrm{n}=71)$ and carrying out daily activities $(18 \%$, $n=72$ ).

Table 2. Types of information pregnant women need

\begin{tabular}{lrr}
\hline \multicolumn{1}{c}{ Types of information } & Frequency & $\begin{array}{r}\text { Percentage } \\
(\boldsymbol{\%})\end{array}$ \\
\hline Pregnancy complications & 223 & 55.75 \\
Delivery complications & 216 & 54.00 \\
Methods of child delivery & 208 & 52.00 \\
Special tests & 193 & 48.25 \\
Taking medicines & 191 & 47.75 \\
Vaccination & 188 & 47.00 \\
Symptoms of pregnancy & 184 & 46.00 \\
Nutrition & 176 & 44.00 \\
Development and growth of the fetus & 174 & 43.50 \\
Newborn care & 171 & 42.75 \\
Items to be taken to hospital for & 158 & 39.50 \\
childbirth & & \\
Family planning & 113 & 28.25 \\
Sexual activity & 87 & 21.75
\end{tabular}




\begin{tabular}{lll} 
Postpartum care (Care of mother) & 85 & 21.25 \\
Breastfeeding & 76 & 19.00 \\
Carrying out daily activities & 72 & 18.00 \\
Physical and psychological changes & 71 & 17.75 \\
$\quad$ after delivery & & \\
Exercise & 69 & 17.25 \\
\hline
\end{tabular}

Source: Survey data

Participants were requested to select sources of information they used when there was an information need. They were given a set of information sources to be selected. The study considered the main sources of information that pregnant women relied on (Table 3). Results revealed that the main source of information was family members and friends. It was followed by midwives $(61 \%, \mathrm{n}=243)$; then doctors $(49 \%)$ and other pregnant women $(49 \%)$. Twenty-nine (29\%) of the respondents reported that they used the internet as one of the sources to get pregnancy-related information. Television \& radio and health programs played the least role in providing information to pregnant women in this study. But in another study, Kamali et al., (2017) identified $60 \%$ of participants relied on mass media. Overdependence on family members and friends is not a healthy indicator in providing information sources.

Table 3. Sources of information used by pregnant mothers during the pregnancy period

\begin{tabular}{lrr}
\hline \multicolumn{1}{c}{ Sources of information } & Frequency & Percentage \\
\hline Family members/Friends & 253 & $63 \%$ \\
Midwives & 243 & $60.8 \%$ \\
Doctors & 194 & $48.5 \%$ \\
Other pregnant mothers & 194 & $48.5 \%$ \\
Internet & 117 & $29.3 \%$ \\
Newspaper & 71 & $17.8 \%$ \\
Books /Journals & 61 & $15.3 \%$ \\
Television/Radio & 57 & $14.3 \%$ \\
Health programs & 56 & $14 \%$ \\
\hline
\end{tabular}

Source: Survey data 
The study was planned to identify the barriers encountered by pregnant mothers in accessing information, in order to break down the barriers with a view to improving the accessibility of information. Figure 1 depicts the results of the study with regard to the barriers they experienced. It reveals that the non-availability of relevant information was the main barrier in accessing the information. It was followed by language $(57.5 \%, \mathrm{n}=230)$, the financial status of the family $(43 \%, \mathrm{n}=172)$, and the educational level of the pregnant mothers $(35.8 \%, \mathrm{n}=142)$. The attitude of the health professional was the least affecting barrier (about 6\%) among the listed barriers in accessing the information. The findings clearly show that pregnant mothers have a positive attitude towards health professionals especially doctors and midwives regarding accessing health information.

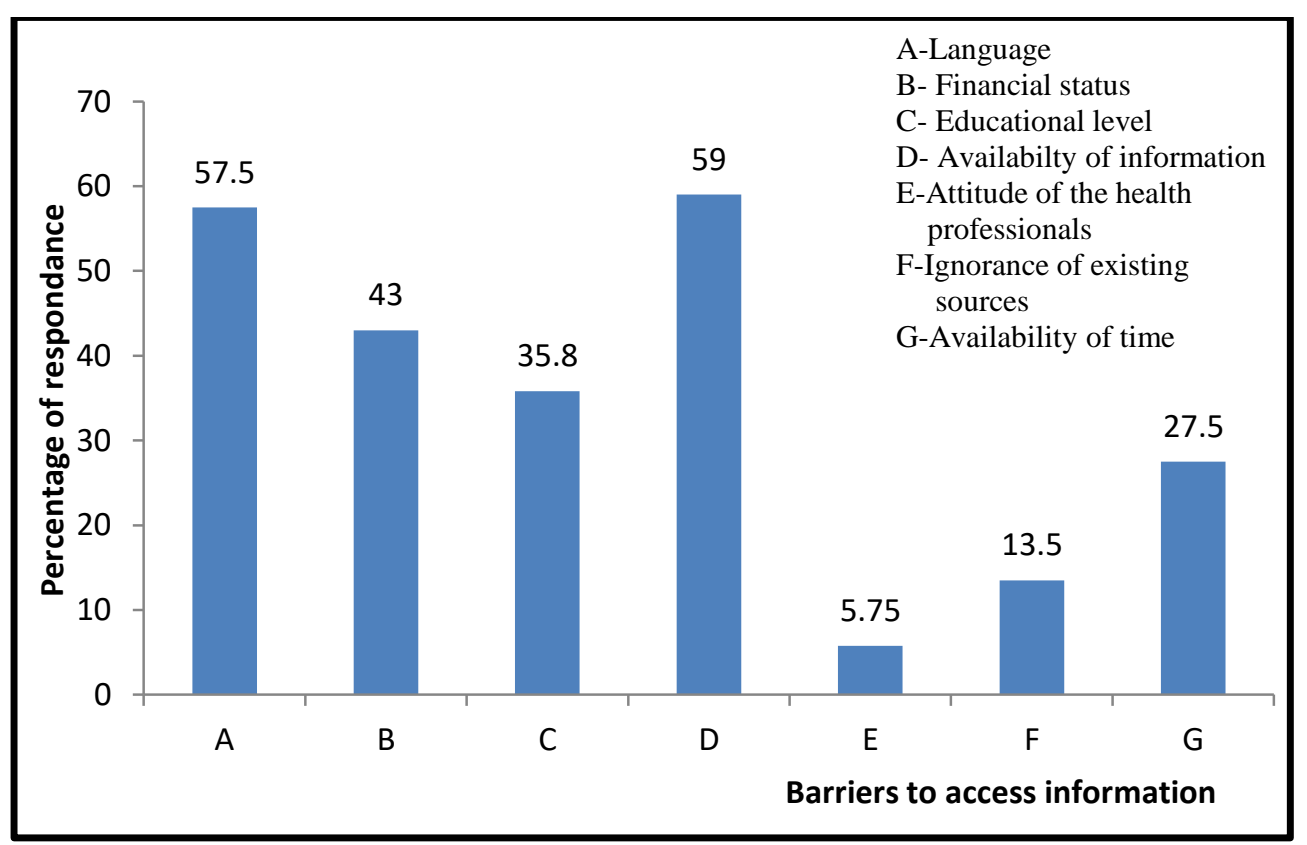

Figure 1. Barriers to accessing the information

In order to analyze the association between age group of pregnant mother and the information sources they preferred, the correspondence analysis was used. The correspondence analysis is similar to chi-squared test but it is often used for high dimensional contingency tables. The results revealed that there was a significant association between age group and the information sources 
they preferred $\left(\chi^{2}=57.8, \mathrm{p}=0.03\right)$. The two-dimensional solution was appropriate to explain the association between variables and the total inertia explained by two-dimensional solution was $73.21 \%$. The results revealed that the age groups of 21-25 and 26-30 and the information sources such as the internet, books, television, newspaper and health programs were significant.

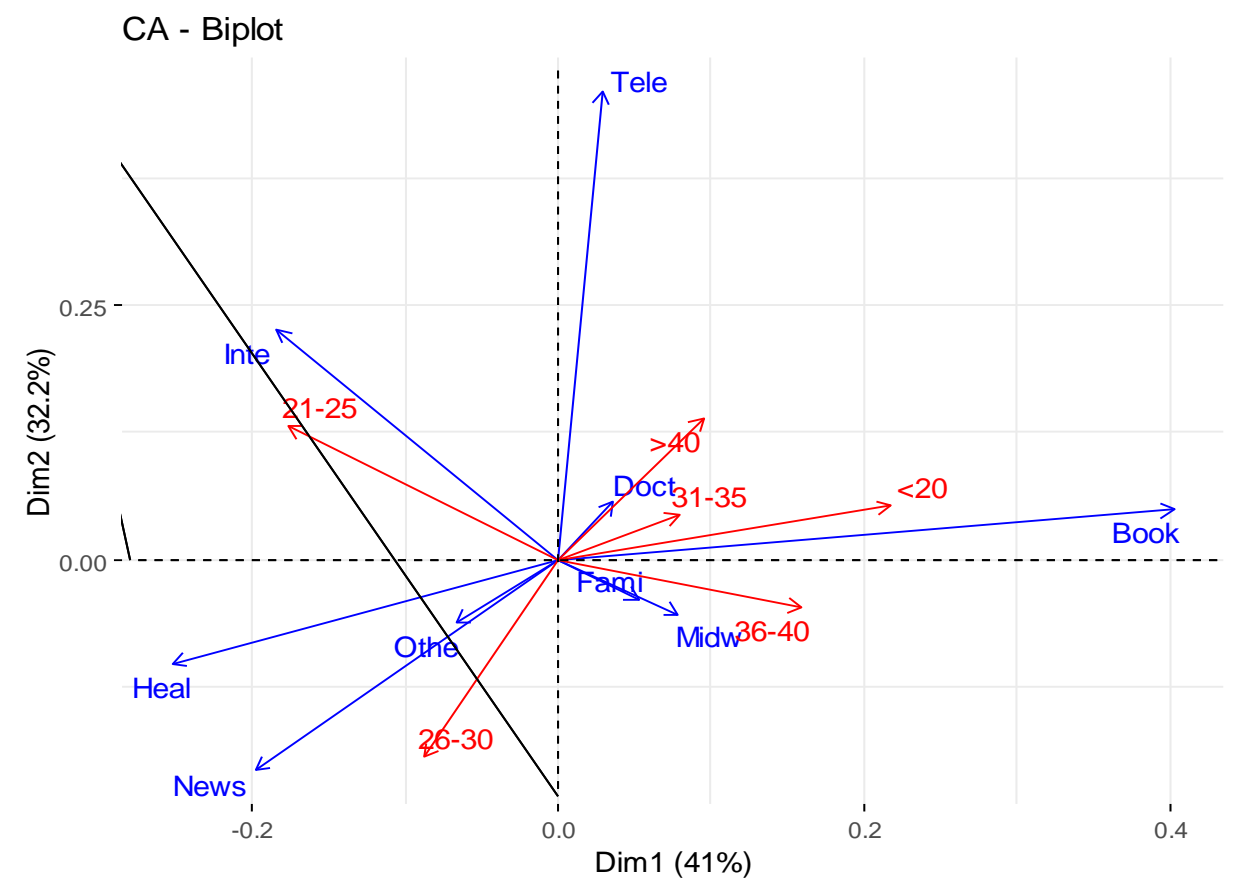

Figure 2. Association between the age group and the preferred information sources

(Information sources - Fami-Family members/friends, Othe-Other pregnant mothers, DoctDoctors, Midw-Midwifes, Inte-Internet, Book-Books/Journals, Heal-Health programs, Teletelevision/radio and News-Newspapers)

(Age groups: <20 - Less than 20 years; 21-25 years; 26-30 years; $31-35$ years; $36-40$ years; >40- Above 40 years)

Figure 2 clearly explains the association between the age group and the information sources they preferred. According to the bi-plot of correspondence analysis, the age group of 21-25 strongly associated with the internet and weakly associated with health programs and, newspapers but 
negatively associated with books. The age group of 26-30 mostly associated with health programs and newspapers but weakly associated with internet, books\& journals and radio \& television. The age group of 36-40 is closely associated with midwives and negatively associated with television \& radio and the internet.

The correspondence analysis also showed that there was a significant association between the educational level of mothers and the information sources they preferred $(\chi 2=96.3, p=0.00)$. The two-dimensional solution was appropriate to explain the association and the total inertia explained by twodimensional solution was $98.6 \%$. The results revealed that the educational levels such as primary education (< Grade 5),up to advanced level and graduates and information sources such as internet, newspapers, books \& journals, doctors, television $\&$ radio and health programs were significant.

Figure 3 indicates the association between the educational level of mothers and the information sources they used to get information during the pregnancy period. Primary education less than grade 5 strongly associated with doctors, but negatively associated with books \& journals, internet and newspapers. Educational level up to advanced level associated with internet, health programs, and television \& radio. The graduates negatively associated with health programs and television \& radio and weakly associated with books $\&$ journals, newspapers, doctors and the internet.

However there was no significant relationship between the family structure and the information sources they often used. The chi square of independence between the two variables is equal to $9.767982(\mathrm{p}$-value $=0.28)$. The results of the correspondence analysis reflect that the pregnant mothers cannot be considered as a homogenous group in accessing different information sources. Different age groups and educational levels have different orientations towards information sources. For example, youngsters are heavily oriented towards web based content, while older people associated with midwives. Therefore, the health programs alone are insufficient in order to provide better information, parallel to such programs and it is vital to publish health information related to pregnancy in different media. 


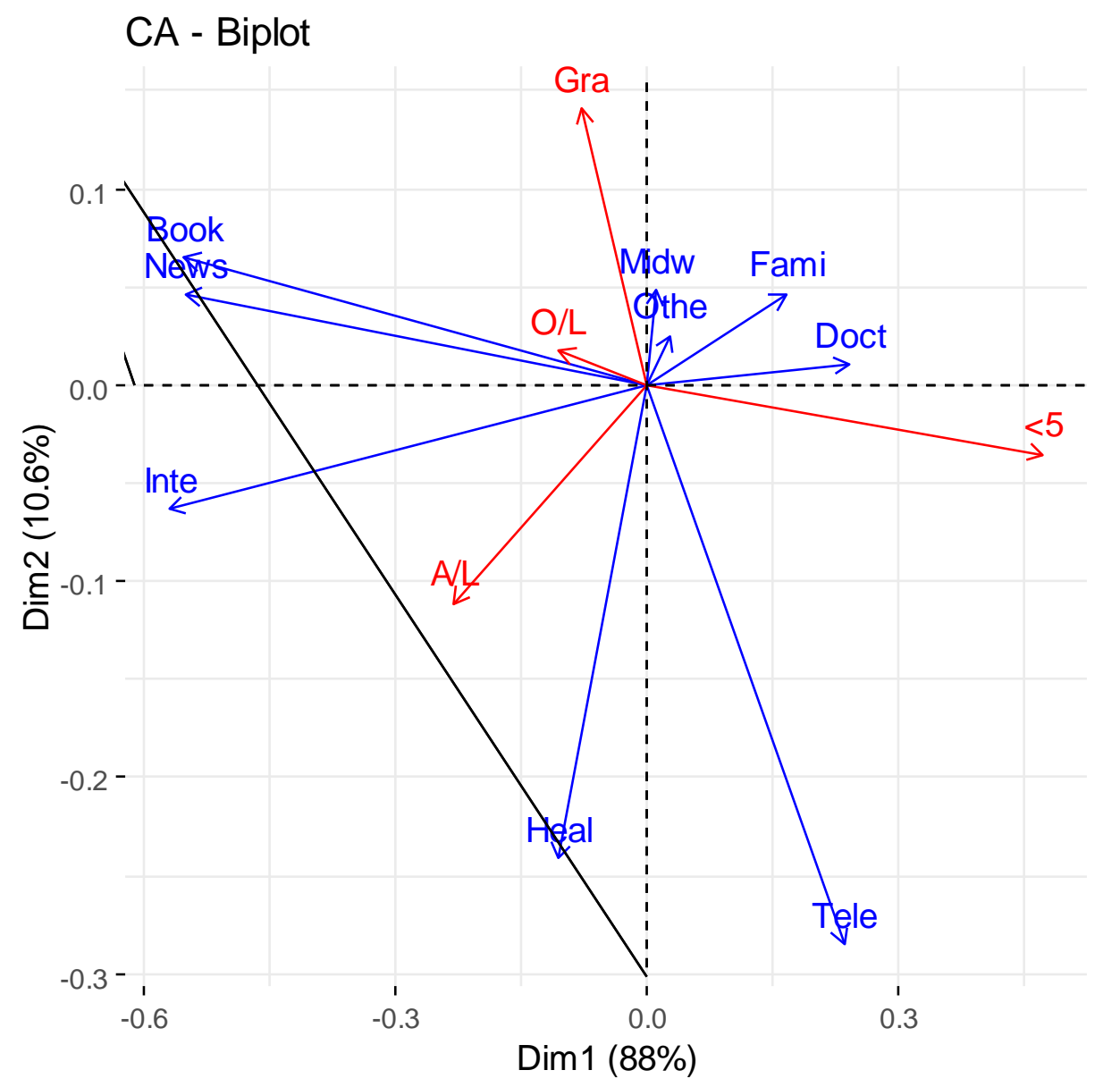

\section{Figure3. Association between the educational level of mothers and preferred information sources.}

(Information sources- Fami-Family members/friends, Othe-Other pregnant mothers, DoctDoctors, Midw-Midwifes, Inte-Internet, Book-Books/Journals, Heal-health programs, TeleTelevision/radio and News-Newspapers)

(Educational levels-<5 -Primary education/L-Ordinary level; A/L-Advanced level; GraGraduate)

The study endorsed that pregnant women actively searched for information. Two third of the participants in the study reported that they searched for information either always or often. Pregnant women showed great interest in searching for information on pregnancy complications (56\%) and delivery complications (54\%). Exercise during pregnancy (17\%) was the least needed 
information among the participants. But, in another study by Shieh, MC Daniel \& Ke (2009) revealed that exercise during pregnancy was one of the often needed types of information among the respondents. It indicates that the importance of exercise during pregnancy must be emphasized among the pregnant mothers in the local community. They used different sources of information. Among all the given information sources, mostly used main source of information was family members and friends. It clearly showed that educational level of family members and friends will directly affect the health information needs of mothers. The traditional practices and beliefs with regard to pregnancy and child birth have shown lapses in the practices of health professionals. Professional support by the public health midwives with regard to providing information was well appreciated by the pregnant mothers. As midwives were the direct mediators in providing information to the mothers in this study, they should be well educated with current updates of pregnancy related health information. Regular training programs should be conducted to update their knowledge. The study revealed that mothers didn't show much interest on mass media to get health related information among the given information sources. Only $15 \%$ of the participants used books and journals. Singh, (2002) found in his study that most popular information source was 'The pregnancy book' which was provided to pregnant mothers in UK funded by the government. Language and the unavailability of information were the main barriers in accessing information pointed out by the participants, since most commonly available books, journals and web based information are published in English language. There is an urgent need to publish books in their native language in order to easily access information.

\section{Conclusion \& Recommendations}

The study shows that pregnant women attending antenatal clinics of Jaffna teaching hospital actively search health information during pregnancy period. It highlighted that the mostly needed information were pregnancy complication, delivery complication, methods of child delivery and special tests conducted during pregnancy. Pregnant mothers used various types of information sources to acquire information. The study pointed out that the information source often used by the majority of participants was traditional sources, which were family members and friends. The role of midwives 
played a major role in providing health information to pregnant mothers. Internet was also identified as one of the source of information used by one third of the participants. Language barrier, caused by insufficient information in their mother tongue and the unavailability of health information were the major restricting barriers in accessing information. It is suggested that small booklets in their mother tongues covering commonly needed important health information be compiled and distributed among all pregnant mothers.

The demographic factors such as age group of participants and the maximum educational level of participants were significantly associated with the types of information sources they used to get health related information during pregnancy. As internet was identified as an important source of information for the pregnant mothers, websites can be developed by the responsible authorities such as universities and regional division of health services to satisfy the information needs of the pregnant mothers especially in that region in Sinhala and Tamil languages. Information can be given in the form of frequently asked questions and answers, which makes it easy to find the solutions for their problems. There can be online forums to freely leave their questions. These questions can be regularly attended and reliable information could be provided as answers be replied within 24 hours' time period. Informative health educational videos can be displayed in the antenatal clinics So that pregnant women can watch it during the waiting time to consult the doctors. Pregnant mothers could read the useful health related books and journals in the clinics, if there is a book cupboard or bookshelf organized in clinics. The findings of the study would be useful in developing and providing collections of appropriate information sources including web based information improving the effectiveness of the programs of support. It will lead to provision of better healthcare during pregnancy.

\section{References}

Aaronson, L.S., Mural, C.M. and Pfoutz, S.K. (1988). Seeking information: Where do pregnant women go? Health Education Quarterly, 15(3), 335345 .

Almoajel, A. and Almarqabi, N. (2016). Online Health-information Seeking Behavior among Pregnant Women in Prenatal Clinics at King Saud 
Medical City, Riyadh. Journal of Womens Health, Issues and Care, 5:3. doi:10.4172/2325-9795.1000228

Bianchi, C.M., Huneau, J.F., Le Goff, G., Verger, E.O., Mariotti, F. and Gurviez, P. (2016). Concerns, attitudes, beliefs and information seeking practices with respect to nutrition-related issues: a qualitative study in French pregnant women. BMC pregnancy and childbirth, 16 (1), 306.

Cochran, W.G. (2007) Sampling Techniques. John Wiley \& Sons, Hoboken.

Davies, M.M. and Bath, P.A. (2002). Interpersonal sources of health and maternity information for Somali women living in the UK: Information seeking and evaluation. Journal of Documentation, 58(3), 302-318.

Durham, G.(1988). A study on the information needs of pregnant women in New Zealand. Australian and New Zealand Journal of Public Health, 12(1), 1-8.

Grimes, H.A., Forster, D.A. and Newton, M.S.(2014).Sources of information used by women during pregnancy to meet their information needs. Midwifery, 30(1), 26-33. http://dx.doi.org/10.1016/j.midw.2013.10.007

Kamali, S., Ahmadian, L., Khajouei, R. and Bahaadinbeigy, K. (2017). Health information needs of pregnant women: information sources, motives and barriers. Health Information \& Libraries Journal, DOI: 10.1111/hir.12200.

Onuoha, U.D. and Amuda, A.A.(2013). Information seeking behavior of pregnant women in selected hospitals of Ibadan Metropolis. Information Impact: Journal of Information and Knowledge Management, 4(1), 76-91.

Singh, D. and Newburn, M. (2000). Access to maternity information and support. The needs and experiences of pregnant women and new mothers. London: National Childbirth Trust.

Shieh, C., McDaniel, A., \& Ke, I. (2009). Information-Seeking and its Predictors in Low-Income Pregnant Women. Journal of midwifery \& women's health, 54(5), 364-372. https://doi.org/10.1016/j.jmwh.2008.12. 017

Song, H., Cramer, E.M., McRoy, S. and May, A. (2013). Information needs, seeking behaviors, and support among low-income expectant women. Women \& health, 53(8)., 824-842 
Wilson, T. D. (1999). Models of information behavior research. Journal of Documentation, 55(3), 249-270.

Wilson, T.D. (2000). Human information behavior. Informing science, 3(2), 49-56. 